\title{
Metafore, allegorie e altre trasformazioni: Quintiliano interprete di Orazio (sul carme 1.14, con alcune osservazioni riguardo alle navi di Virgilio e Ovidio)*
}

\author{
Andrea Cucchiarelli \\ Sapienza - University of Rome
}

Nella sua trattazione di quel particolare tropo che è l'allegoria, nel libro VIII dell'Institutio oratoria, Quintiliano fa riferimento al carme 1.14 di Orazio e alle Bucoliche di Virgilio (in particolare l'ecloga 9). Questi due riferimenti, che si spiegano e giustificano all'interno di una institutio volta a formare un abile retore, hanno rappresentato per secoli, già a partire dall'età antica fino all'età moderna, l'interpretazione standard dei due testi di Orazio e di Virgilio. Ciò anche in conseguenza, come è naturale, della grande autorità scolastica di Quintiliano. Vale la pena di rileggere con attenzione il luogo quintilianeo:

Rielaboro qui la relazione tenuta al convegno di San Paolo, che è stato una preziosa occasione di incontro e riflessione: ringrazio gli organizzatori e i partecipanti tutti, alcuni dei quali sono stati particolarmente generosi di osservazioni (si vedano infra le nn. 16 e 24). Desidero inoltre ringraziare Giuseppe Lentini per aver discusso con me di alcune questioni "nautiche" (alcaiche e non). Le pagine che seguono conservano alcune tracce di oralità, che spero al lettore non riescano troppo sgradevoli. 
allegoria, quam inversionem interpretantur, aut aliud verbis, aliud sensu ostendit, aut etiam interim contrarium. Prius fit genus plerumque continuatis translationibus ut 'O navis, referent in mare te novi / fluctus: o quid agis? fortiter occupa / portum', totusque ille Horati locus, quo navem pro republica, fluctus et tempestates pro bellis civilibus, portum pro pace atque concordia dicit. [45] tale Lucreti 'avia Pieridum peragro loca', et Vergili 'sed nos immensum spatiis confecimus aequor, / et iam tempus equum fumantia solvere colla'. [46] sine translatione vero in Bucolicis: 'certe equidem audieram, qua se subducere colles / incipiunt mollique iugum demittere clivo, / usque ad aquam et veteris iam fracta cacumina fagi, / omnia carminibus vestrum servasse Menalcan'. [47] hoc enim loco praeter nomen cetera propriis decisa sunt verbis, verum non pastor Menalcas, sed Vergilius est intellegendus.

L'allegoria, che si traduce con il termine 'inversione', o mostra una cosa con le parole, un'altra con il senso, oppure significa anche, talvolta, il contrario. Il primo genere è dato per lo più da una successione di metafore, come « $\mathrm{O}$ nave, $\mathrm{ti}$ riporteranno in mare nuovi / flutti: ma che fai? forza, entra / in porto», e tutto quel famoso luogo di Orazio, in cui il poeta intende la nave per la repubblica, i flutti e le tempeste per le guerre civili, il porto per la pace e la concordia. [45] Così Lucrezio "percorro i remoti luoghi delle Pieridi» e Virgilio «ma noi abbiamo concluso un'immensa distesa / ed è tempo ormai di sciogliere ai cavalli il collo fumante». [46] Senza metafora, invero, l'allegoria compare nelle Bucoliche: «certo, io avevo sentito, là dove i colli / cominciano a scendere e a piegarsi in dolce pendio, / fino all'acqua e ai vecchi faggi, ormai cime spezzate, / che tutto il vostro Menalca avesse conservato grazie ai canti». [47] In questo luogo, infatti, tutto il resto, tranne il nome, è definito da termini propri, ma in realtà si deve intendere che non si tratta del pastore Menalca, ma di Virgilio. ${ }^{1}$

1 Quint., Inst. 8.6.44-47. 
Dunque, Quintiliano definisce un primo genere di allegoria, consistente in una successione continuata di metafore (continuatis translationibus), privo cioè di qualsiasi elemento, per così dire, 'extra-allegorico', il quale, facendo riferimento al significato reale, sveli il gioco. Ad esemplificare la definizione il primo riferimento che Quintiliano va a scegliere è il carme oraziano, la cui conoscenza egli dà certo per scontata tra i suoi allievi e lettori (totusque ill e Horati locus). Seguono due più rapidi esempi tratti da due altri testi assai noti, il De rerum natura e le Georgiche, in cui, naturalmente, lo spazio percorso (peragro loca; confecimus aequor) è quello, metaforico-allegorico, della poesia (Lucr. 4.1 = 1.926; Verg., Georg. 2.541-2). Ultimo esempio tratto dalla poesia, prima che Quintiliano passi alla prosa, per la quale fa riferimento ad un'opera perduta di Cicerone, è quello dell'ecloga nona (vv.7-10), in cui appunto Menalca non sarebbe altro che allegoria di Virgilio: verum non pastor Menalcas, sed Vergilius est intellegendus.

In un contesto in cui si voglia riflettere sul tema di New trends and revaluations, cioè Nuove tendenze e rivalutazioni (ovvero nuove valutazioni) nella poesia augustea, fortunatamente non si avverte il bisogno di riaprire la discussione sulla allegoricità della poesia bucolica virgiliana. Qui il retore Quintiliano è buon testimone dell'antica tradizione esegetica secondo cui il testo bucolico presenterebbe una precisa identificazione allegorica tra Virgilio e i suoi personaggi (Menalca, nella fattispecie). Da questa tradizione esegetica, perdurante lungo tutto il Medioevo e l'età moderna, è derivata, soprattutto all'interno della grande stagione critica Otto-Novecentesca (più o meno consapevolmente post-romantica) una certa svalutazione della poesia virgiliana che, rispetto al modello teocriteo, si concederebbe ad un gioco intellettualistico, tutt'altro che spontaneo, di travestimenti e allusioni. $\mathrm{Ma}$, va ribadito, non è il caso di insistere oggi sul fatto che le allusioni alla realtà contemporanea delle confische, che certo interessarono più o meno direttamente anche il poeta, sono 
soltanto una componente tra le tante di una poesia che mira ad una visione della storia di ben più ampio respiro ${ }^{2}$. Sarebbe opportuno, in realtà, tentare una riflessione complessiva, che fosse anche una sorta di bilancio critico, sulla reale incidenza dell'allegoria nell'opera virgiliana, a partire dalle Bucoliche. $\mathrm{Ma}$ in questo contributo ci concentreremo su Orazio, la cui interpretazione sembra necessitare di un più urgente correttivo.

\section{Quale allegoria per la nave di Orazio? (Quintiliano va sicuramenterelativizzato)}

Se, dunque, l'interpretazione quintilianea, nel caso di Virgilio, può essere oggi serenamente relativizzata come appartenente ad un preciso contesto storico e culturale, ormai lontano, nel caso del carme oraziano, ben diversamente, Quintiliano è ancora a fondamento dell' interpretazione corrente, come si può facilmente osservare consultando i commenti di riferimento al libro I dei Carmina ${ }^{3}$. Dunque, stando a Quintiliano, qui Orazio si rivolgerebbe, esortandola ad entrare in porto, ad una nave piuttosto malridotta, in cui andrebbe riconosciuta la respublica, mentre flutti e tempeste starebbero per la guerra civile e il porto, invece, per la pace e la concordia.

2 Basti ricordare come si esprime uno tra i più influenti commentatori delle Bucoliche, Coleman (1977, 274-5), che pure non si distingue certo per l'eccessivo scetticismo a riguardo: "Clearly Vergil's own experience provided much of the inspiration in both poems [scil. ecl. 1; 9]; but his chief concern is once again a more general one etc.»; ancor più reciso Clausen $(1994,271)$ ad 9.10: «Menalcas is a general benefactor». È noto che la tradizione biografica virgiliana, con le sue implicazioni esegetiche, è attualmente vagliata con occhio assai critico dagli studiosi, come ben si può vedere dalla trattazione di Horsfall (1995).

3 Con la menzione di Quintiliano si apre l'introduzione al carme di Nisbet; Hubbard (1970, 179), che in seguito si esprimono più d'una volta in termini di 'Ship of State' (pp. 180-181); l'interpretazione quintilianea è, in sostanza, fatta propria ancora da Mayer (2012, 136-7) (dove è definita, significativamente, come lo 'standard': spec. p. 136); ma cfr. già, come esempio assai autorevole e influente, Kiessling; Heinze $\left(1960^{10}, 71\right)$. 
Così, appunto, la decodificazione quintilianea: navem pro republica, fluctus et tempestates pro bellis civilibus, portum pro pace atque concordia.

Diciamo subito che l'interpretazione di Quintiliano è nella sostanza corretta, nel senso che stabilisce giustamente la natura allegorica del carme in un quadro interpretativo che è quello p o 1 i t i c o. Ma la facile generalizzazione 'Nave dello Stato' ovvero 'Ship of State' (navis = respublica), se poteva bastare a Quintiliano e ai suoi allievi, interessati all'impiego pratico di una comoda allegoria in contesto retorico, non dovrebbe bastare agli interpreti oraziani. Non si può pretendere da Quintiliano, che citava presumibilmente a memoria i testi poetici (talvolta, a quanto pare, con qualche inesattezza ${ }^{4}$, e unicamente in funzione dei propri scopi didattici, la precisione e gli interessi dell'esegeta. E infatti, a dimostrazione della sua problematicità, l'equivalenza nave $=$ respublica ha prodotto due conseguenze molto diverse, ma pure, in fondo, analoghe: da un lato la svalutazione estetica del carme, dall'altro l'idea che qui l'allegoria non sia di ambito politico ma di ambito erotico. Due conseguenze estreme, si potrebbe dire eccentriche (e, aggiungerei, insoddisfacenti), di quello che è, in effetti, un reale problema.

Della prima conseguenza dà buona testimonianza un fondamentale commento al libro I dei Carmina, quello di Nisbet; Hubbard $(1970,181)$ dove si legge: «Horace ode, though elegant as always, is less than a masterpiece. The poet's immediate impulse was not a worsening political situation (which is perhaps why the date is so uncertain), but a perverse determination to write allegory. Alcaeus knew what was like to be swept along in a black boat [...]. Horace can supply only a

4 Un caso in cui, probabilmente, la memoria ingannò Quintiliano è carm.1.12.41 incomptis ... capillis (che egli in inst. 9.3.18, cita con intonsis); sulla non totale affidabilità delle citazioni quintilianee giunse a conclusioni condivisibili già Cole $(1906,51)$ spec. 51; cf. anche Odgers $(1933,186)$. Questi studi, ancora validi e utili, andrebbero però, senza dubbio, aggiornati. 
civil servant's inventory of the damage. His personification of the ship is stereotyped and unconvincing, more so than in I. 3". Nella citazione l'idea dell'allegoria oraziana come astrazione intellettualistica si salda, evidentemente, al (pre-)giudizio già romantico (e non solo romantico) di Orazio poeta freddo e mediato, rispetto al suo predecessore greco, più spontaneo e immerso nella realtà di ciò che descrive ${ }^{5}$.

La seconda conseguenza è legata ad un brillante articolo di W. S. Anderson ${ }^{6}$, che ha avuto l'indubbio merito di osservare una serie di incongruenze da cui l'interpretazione della nave in termini di astrazione onnicomprensiva (la 'Nave dello Stato') è resa quanto meno disagevole: di qui l'interpretazione della nave come allegoria di una donna che il poeta avrebbe amato, almeno in passato, ma che ora farebbe bene, in ragione dell'età matura, a ritirarsi dalle tempeste dell'amore (e, forse, a ritornare dal suo amante, Orazio stesso). Delle incongruenze notate da W. S.

5 È ben probabile che in Nisbet e Hubbard agisca direttamente l'influente giudizio di Fraenkel $(1957,157)$, che, aprendo la trattazione di Carm. 1-3 proprio con 1.14, scriveva: "O navis referent is certainly not one of Horace's masterpieces"; cf. l'ediz. ital. $(1993,217)$; a sua volta Fraenkel si poneva sulla linea della grande filologia tedesca, in particolare il Wilamowitz (1913), per quanto dal Wilamowitz qui Fraenkel prendesse le distanze riguardo alla questione, che egli giudicava evidentemente futile (ma che non lo è affatto!), se Orazio si ritragga o meno a bordo della nave: cf. $(1957,157$, n. 2.). Vale la pena citare lo stesso Wilamowitz, perché qualcosa delle sue parole sembra risuonare ancora (nonostante Fraenkel, nello specifico!) nel comm. di Nisbet; Hubbard: "Denn er [scil. Horaz] steht am Ufer und sieht das Schiff im Kampfe mit den Wellen, Alkaios fährt darauf und besteht die Gefahr» (1913, p. 312). Giova, a questo punto, ricordare come si esprimesse a suo tempo G. Pasquali, quando reagiva al «pregiudizio, qualche anno fa ancor più diffuso che non ora, che Orazio fosse un Alcaeus dimidiatus come Virgilio un dimidiatus Homerus" (1964 [1920], 18).

6 Anderson (1966, 84-98); la tesi di Anderson ha trovato non pochi riscontri, a partire già da Shackleton Bailey $(1982,89)$, che mostra di prenderla in seria considerazione; cf. inoltre Knorr (2006); Kruschwitz (2007). Non più di un rapido riferimento all'articolo di W. S. Anderson si legge in Nisbet; Hubbard cit., che si esprimono a riguardo in termini di «strange theories» (p. 180): non è forse ozioso notare che W. S. Anderson nel suo articolo si era mostrato assai critico, seppure rispettosissimo, nei confronti di E. Fraenkel. 
Anderson, e da altri dopo di lui, mi limito per ora a ricordarne una sola: come potrebbe il poeta nella quinta e conclusiva strofa rivolgersi alla Nave dello Stato come ad un'entità distinta da sé, verso la quale egli avrebbe provato prima sazietà/disgusto e poi desiderio? Come ci si può estraniare dallo Stato, di cui, per definizione, ogni cittadino fa parte?

$\mathrm{Ma}$ è arrivato il momento di ascoltare la voce di Orazio stesso, con i suoi dinamici asclepiadei (il sistema metrico del carme 1.14 è il cosiddetto terzo asclepiadeo):

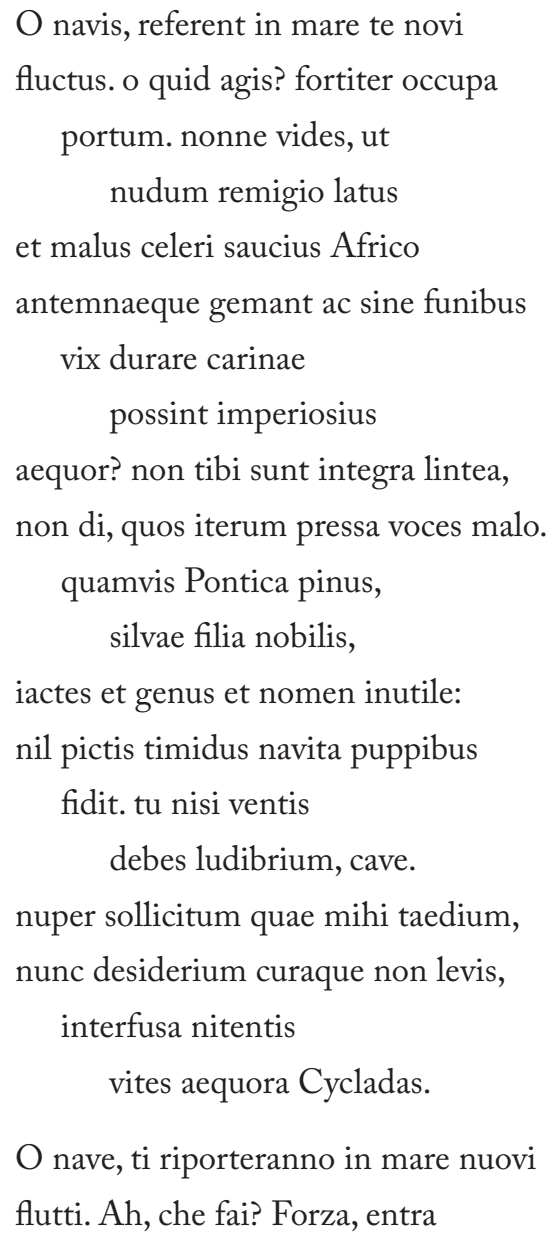

$\mathrm{O}$ nave, ti riporteranno in mare nuovi

flutti. Ah, che fai? Forza, entra 
in porto! Non vedi forse, come

la fiancata sia priva di remi

e l'albero ferito dal veloce Africo,

come le antenne gemano e senza le funi

a mala pena la chiglia resistere

possa ad un così imperioso

mare? Non hai integre le vele,

non gli dèi, che tu, premuta dal male, di nuovo possa

invocare.

Per quanto tu, fatta di pino del Ponto,

figlia di nobile selva,

ti vanti della tua origine e del tuo nome inutile,

il marinaio, se ha paura, di poppe dipinte

non si fida. Tu, se dei venti

non vuoi divenire trastullo, sta' attenta.

Di recente tu mi sei stata affannoso disgusto,

ora mi manchi, mia angoscia non lieve:

che tu possa evitare il mare che sta

tra le Cicladi splendenti. ${ }^{7}$

Del carme 1.14 mi sono occupato una decina di anni fa e sono tornato ad occuparmi recentemente all'interno di uno studio sul mare (anche non-allegorico!) in Orazio ${ }^{8}$. Mi rendo conto di quanto sia difficile mettere in discussione uno stereotipo così radicato nell'insegnamento scolastico, di Orazio e non solo, quale è la 'Nave dello Stato', che tanta fortuna, anche attraverso Quintiliano, ha avuto nella cultura occidentale.E non escluderei che l'immagine di un Orazio che si rivolga, indistintamente, ad un'entità astratta come la respublica Romana possa riuscire

\footnotetext{
Hor., Carm. 1.14

8 Cf. Cucchiarelli (2004); (2005); Cucchiarelli (2015a, spec. 301-317).
} 
confortevole e rassicurante, in linea con l'interpretazione generale di un Orazio non troppo politicamente esposto (e quindi un poco vago e indistinto), nel male come nel bene' .

Proprio il fatto di aver già discusso la questione in dettaglio altrove mi permette, spero, di essere piuttosto sintetico. Dunque, mi limiterò ad elencare quelli che ritengo i punti fondamentali per arrivare ad una corretta interpretazione del carme oraziano, qui e là concedendomi qualche breve osservazione di sostegno $\mathrm{o}$ in aggiunta rispetto a quanto ho già avuto modo di osservare in passato. Nel passaggio dall'uno all'altro punto (per un totale di otto) chiarirò progressivamente quella che ritengo essere la giusta interpretazione del carme.

1) Il modo in cui Orazio si esprime, soprattutto nell'ultima strofe, non si adatta ad una nave intesa come astrazione onnicomprensiva, quella dello Stato-respublica. Orazio parla come se non fosse a bordo della nave, ma ciò sarebbe impossibile, o quanto meno molto difficile, se egli si stesse riferendo allo Stato, di cui naturalmente è parte. Termini come desiderium e cura appartengono ad un lessico personale, dell'amore/ amicizia, adatto piuttosto ai rapporti con un individuo o un gruppo di individui, ma non del tutto appropriato e consono allo Stato-respublica.

2) Quella di Quintiliano non è l'unica interpretazione antica del carme. Sia Porfirione che il cosiddetto Pseudo-Acrone (interpreti, si noti, istituzionalmente più vicini al testo oraziano rispetto a Quintiliano) testimoniano il tentativo di identificare, all'interno dell'allegoria politica, un preciso riferimento ad un definito individuo, che si tratti di Bruto o di Sesto Pompeo.

\footnotetext{
9 Si veda in proposito Clay $(2010,139-40)$, che, infatti, si dichiara non convinta dalle argomentazioni da me avanzate nell'articolo del 2004-2005 (n. 41, a p. 145).
} 
Ecco i due luoghi dei commentatori ${ }^{10}$ (si noti che in Ps.Acrone l'interpretazione navis = respublica è liquidata piuttosto rapidamente come una possibilità tra le altre):

in hac ode ad Marcum Brutum loquitur, qui apud Philippos Macedoniae urbem ab Augusto fusus videbatur rursus se instruere ad pugnam. Merito autem poeta per allegoriam metuere se pro eo testatur, quoniam sub ipso militaverat. Qui tamen ex proelio Philippico fatigatus, receptus ab Augusto veniam ab eo meruit. ${ }^{11}$

per allegoriam ode ista bellum civile designat, ut quidam volunt, alii rempublicam. Certius tamen est quod Sextum Pompeium filium Pompei moneat qui, postea quam foedus cum triumviris fecit, bellum civile denuo reparare voluit. Secundum autem civile bellum inter Augustum Caesarem et Cassium et Brutum fuit, qui fuerunt Gai Caesaris interfectores, sub quibus Horatius militavit. ${ }^{12}$

3) La nave, per la sua stessa natura di oggetto collettivo (che presuppone, cioè, un equipaggio) non può essere agevolmente identificata con un solo individuo, che sia Bruto, Sesto Pompeo o altri, ma piuttosto identifica un gruppo, cioè, allegoricamente, una parte politica. Già nei celebri carmi alcaici (spec. frr. 208a; $6 \mathrm{~V}$.) la nave rappresenta lo strumento di aggregazione di un gruppo di compagni (gli hetairoi) che si percepivano, certo, come depositari dei veri valori della polis, ma non potevano propriamente identificarsi con essa ${ }^{13}$. Un concetto come quello quintilianeo di Stato-respublica, molto vicino di fatto al concetto

10 Qui riportati nel testo al momento più affidabile, che è quello curato da M. Spurio (Porfirione) e L. Paretti (Ps.-Acrone), nel III volume dell'Enciclopedia Oraziana (Roma 1998); sull'esegesi antica di 1.14 cf. già Reitzenstein (1918, 393-6).

11 Porph. Ad carm. 1.14.1.

12 Ps.-Acr. Ad carm. 1.14.1.

13 Presuppongo qui il noto lavoro di Rösler (1980). 
moderno, è senz'altro anacronistico per Alceo, ma ancora forse non del tutto appropriato nemmeno per Orazio e la sua epoca.

4) In questa prospettiva si chiarisce non soltanto l'ultima strofa del carme, ma tutta la sua seconda parte (si noti che Orazio ha nettamente bipartito il carme, dedicando alle prime due strofe e mezzo la ripresa di Alceo, alle seconde due e mezzo la codificazione del nuovo e originale messaggio allegorico $\left.{ }^{14}\right)$. La nave che si vanta dei suoi illustri natali (13 iactes et genus et nomen inutile), ma che è nella realtà troppo malridotta per ispirare fiducia, nei confronti della quale Orazio ha provato prima un senso di distacco molto forte, ma comunque angoscioso (17 sollicitum ... taedium), e poi un senso di mancanza e preoccupazione (18 desiderium curaque non levis), va identificata con la parte politica degli oppositori, in cui fu sempre ben netta la componente della grande aristocrazia romana. Erano gli aristocratici della nobilitas ad essere vanamente fieri delle loro origini.

5) Nel contesto della concreta vita politica e militare di Roma, per come fu vissuta in prima persona dallo stesso Orazio, l'imitazione di Alceo perde quel suo aspetto freddo e intellettualistico, si direbbe libresco, che tanto ha nuociuto all'interpretazione del carme. Come Alceo e i suoi compagni, così anche Orazio e tanti altri cittadini di Roma si trovarono a combattere contro altri concittadini, conobbero le durezze del campo di battaglia e anche della vita marinara ${ }^{15}$. Per il poeta Orazio e il suo pubblico, dunque, doveva essere più che evidente quale concreto contesto trovasse una tale imitazione allegorica

14 Su questo vd. infra, p. 161.

15 Come Orazio dice proprio a proposito di Alceo: dura navis, / dura fugae mala, dura belli (Carm.2.13. 27-8). In questo contesto è significativo che già nell'epodo 16 , come strumento di fuga dalle guerre civili, sia presa in considerazione la flotta, su cui il popolo romano viene esortato dal poeta a salire, per lasciare Roma e volgersi verso le utopistiche Isole dei Beati: cf. Epod. 16, spec. 24 ratem occupare quid moramur?; su navi, flotte, dissidi civili, si può vedere Mastrocinque (2016). 
dello stasiotico Alceo: quello della guerra civile, lungamente combattuta, dalle Idi di Marzo e Filippi in poi, per mare e in Grecia. La parola finale del carme, sull'interpretazione della quale si è molto discusso, senza dubbio ha l'effetto di proiettare l'immaginazione del lettore appunto nei mari della Grecia: Cycladas $^{16}$.

6) L'allocuzione di Orazio ad una nave (alcaica) che, nonostante fosse malridotta, voleva continuare ad affrontare i pericoli dei venti, identifica quel partito di irriducibili che, fondandosi su di una forte matrice aristocratica, continuò l'opposizione anche dopo Filippi, prima schierandosi con Sesto Pompeo e poi con Antonio. Qui Orazio non ha bisogno di far riferimento ad un contesto storico preciso, perché quello che gli interessa è definire un paradigma comportamentale e politico che, dopo Filippi, non aveva più senso: quello della ribellione (o, in termini alcaici, stasis). Non è tanto corretto parlare di incertezza nella datazione, come fanno Nisbet; Hubbard («the date is so uncertain»), quanto di deliberata e mirata indeterminatezza, che rende il carme valido e attuale in tutta la situazione politica e militare successiva a Filippi (e, si può aggiungere, anteriore ad Azio). Orazio aveva combattuto a Filippi dalla parte repubblicana, ma poi si era staccato dai suoi,

$\overline{16}$ Proprio le Cicladi si affacciano alla mente di Virgilio nell'iperbolica comparazione che mira ad esprimere le enormi dimensioni delle navi che si scontreranno ad Azio, la battaglia con cui, nella propaganda augustea, le guerre civili si conclusero: Aen. 8.691-2 pelago credas innare revolsas / Cycladas aut montis concurrere montibus altos (l'effetto è anche di suggerire la forza devastante delle guerre civili, capaci di sconvolgere il mondo come in un cataclisma o gigantomachia). Nel convegno di San Paolo Andreas Michalopoulos, considerata la trasparente etimologia di Cyclades (ad es. Plin., Nat. 4.65 in orbem sitae; anche Serv. ad Aen. 3.126; Maltby (1991, 169), s.v. Cyclades), ha osservato che una geografia circolare ('ciclica') è particolarmente adatta al contesto oraziano: l'ultima parola del carme vuole dunque contribuire a suggerire il pericolo della guerra civile, che è 'avvolgente' e in perenne rinnovamento, 'ciclico', appunto (1-2 novi fluctus, all'altro capo del carme) - un pericolo da cui è assai difficile uscire, una volta che si sia commesso l'errore di entrarvi. 
per i quali ora torna a provare un vivo sentimento, che è quello della preoccupazione. Al paradigma della ribellione il carme 1, 14 contrappone quello della resa: una resa decorosa, di chi ha molto combattuto, ma si rende conto che proseguire sarebbe solo un insensato suicidio, un suicidio oltretutto dannoso per l'intera comunità dei concittadini.

7) Lo stesso Orazio riconosce nella nave di Alceo lo strumento della lotta e dell'impegno guerriero, nell'allocuzione al barbitos che riveste un ruolo rilevante nella sequenza finale del libro I: Lesbio primum modulate civi, / qui ferox bello tamen inter arma, / sive iactatam religarat udo / litore navim eqs. (Carm. 1.32, 5-8). Si noti, la nave che Alceo all'occasione sa legare all'umida riva è una nave $\mathrm{s} q \mathrm{u}$ a s s a $\mathrm{t}$ a, evidentemente dalle tempeste di un mare che è anche allegorico, da cui, cioè, è rappresentata potentemente la violenza della stasis.

8) Nell'edizione alessandrina di Alceo, che si impose come normativa nella diffusione tra il pubblico dei lettori ellenistici e quindi romani, i carmi allegorici ricevevano la loro soluzione interpretativa sia da appositi sussidi esegetici (come i commentari) sia dal fatto di essere collocati all'interno dei libri stasiotici. Risultava evidente dalla loro stessa collocazione editoriale che i carmi della nave avevano un significato politico, giacché essi seguivano o precedevano carmi in cui l'argomento stasiotico era esplicito. In primo luogo, dunque, osserverei - anche se non intendo insistere troppo su questo punto - che nella raccolta oraziana il carme 1.14 è seguito dal carme 1.15 Pastor cum traheret, in cui non pochi interpreti hanno ravvisato la proiezione mitico-omerica della vicenda di Antonio e Cleopatra: dunque, un tema che rimanda alle guerre civili, nella loro fase ultima e decisiva. Ma credo che, affidandosi all'insieme della propria raccolta lirica, Orazio abbia in effetti voluto indirizzare i lettori esperti (che, cioè, conoscono l'insieme della poesia lirica oraziana nei suoi vari volumina) 
verso la giusta soluzione dell'allegoria. Nel carme 2.7, all'amico Pompeo finalmente rientrato a Roma (forse dopo l'amnistia successiva ad Azio?), Orazio rievoca in nitidi e dignitosissimi versi l'avventura di Filippi. Sono versi veramente molto celebri, ma pure gli interpreti del carme 1.14 non ne hanno tenuto debito conto:

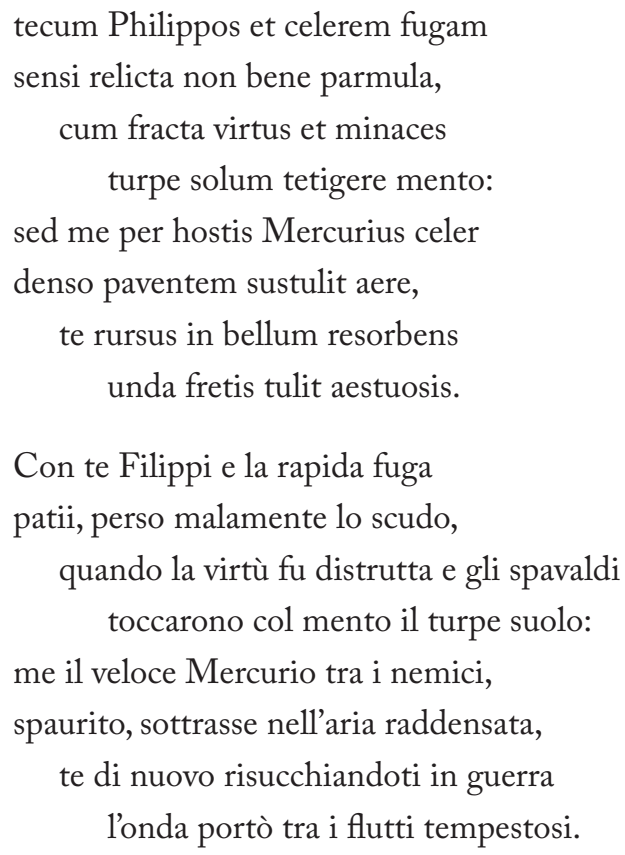

Nel metro più propriamente alcaico, cioè, appunto, la strofe alcaica, Orazio dice come Mercurio lo avesse fortunosamente sottratto alla mischia. I due amici si erano ritrovati entrambi assai a malpartito, dopo aver subito quel terribile colpo capace di annientare qualunque forma di virtus. Entrambi malridotti, dunque, c o m e la nave di 1.14. Ma il solo Pompeo fu risucchiato dall'onda (della guerra civile) tra mari agitati: rursus ... resorbens / unda fretis ... aestuosis (15-16), che è una variazione, oserei dire esplicativa, di 1.14, spec. 1-2 referent ... novi / fluctus; 19-20 interfusa ... / ... aequora Cycladas (qui non 
conta, naturalmente, il rapporto cronologico tra i due carmi, ma la loro connessione sincronica). Così, dunque, i destini dei due amici si divisero, esattamente come sono separati Orazio e la nave in 1,14 . Verso un amico come Pompeo, lungamente e pericolosamente assente da Roma, che cos'altro potrà aver provato Orazio, prima che egli facesse ritorno, se non un sentimento di mancanza e di preoccupazione (desiderium e cura)? In Alceo l'allegoria trova la sua soluzione nel contesto editoriale dell'opera: anche per l'allegoria oraziana vale analogamente il principio aristarcheo di 'chiarire Orazio con Orazio ${ }^{17}$.

Dunque, in sintesi: la nave del carme 1.14 va identificata con la parte degli oppositori, quei repubblicani, spesso di nobili origini o comunque guidati da nobiles, assieme ai quali Orazio aveva combattuto a Filippi. Ma dopo Filippi Orazio si arrese, mentre molti tra i suoi vecchi compagni continuarono la lotta almeno per un decennio: a loro qui Orazio si rivolge.

Non sto ad entrare in questioni di gusto, oserei dire che non è questo il mio compito. Lascio, dunque, al giudizio personale di ognuno la questione sollevata da Nisbet; Hubbard con la loro svalutazione del carme oraziano. E certo Orazio stesso doveva rendersi conto di quanto fosse difficile gareggiare con Alceo. Ma è lecito credere che una così decisa svalutazione del carme sia la diretta conseguenza di un'interpretazione che veda nella nave una generica, direi sbiadita, raffigurazione della

17 Che la guerra civile debba essere immaginata come un grande e trascinante movimento lo dice anche un altro fondamentale luogo oraziano, l'attacco del libro II, nel carme a Pollione, in alcaiche: Motum ex Metello consule civicum / bellique causas eqs. (1-2). Vale la pena di notare che, dopo il carme 2.2 in saffiche a Sallustio, nipote e figlio adottivo dello storico, nel carme 2.3 , di nuovo in alcaiche, Orazio si rivolge a un personaggio fortemente coinvolto nelle varie vicende delle guerre civili, Q. Dellio. Non ci possono essere dubbi sul tono storico-politico del libro II nel suo avvio, che riprende, dopo la parentesi degli erotici carmi 2.4-5 e del carme 2.6 (dove comunque già si affaccia il tema della stanchezza, dopo i viaggi e la militia), con il carme 2.7 . 
respublica tout court. In realtà, come ho cercato di dimostrare, la concretezza descrittiva e immaginativa delle allegorie alcaiche non va del tutto perduta nella reinterpretazione e nuova sintesi di Orazio.

La nave, che in Alceo era simbolo e strumento della lotta politica, principio di aggregazione per il poeta e i suoi compagni, diventa in Orazio allegoria di una partecipazione militante alla lotta civile, da cui il poeta si era distaccato, ma che invece ancora riuniva interi equipaggi di antichi compagni. Non so se si può dire che quella di Orazio fosse una «perverse determination to write allegory» e se una simile perversione sia un genere di peccato che va rimproverato ad un poeta. Ma direi che un'opportuna conoscenza del contesto storico tra il 44 e il 31 a.C. mostra quanto l'autore del carme 1.14 , non troppo diversamente da Alceo, dovesse trovarsi nel vivo di una «worsening political situation»: ancora fino ad Azio (e ancora oltre, in realtà) il pericolo di una ricaduta nella guerra civile era costante (e questo pericolo, certo, era amplificato dagli intellettuali come Orazio vicini al gruppo di potere di Ottaviano, Mecenate, Agrippa $)^{18}$.

18 Su questi argomenti resta fondamentale ediz. ital. a cura di A. Momigliano. Ma si aggiunga anche, in particolare sull'ascesa di Ottaviano nelle prime fasi della sua azione politica, il volume di Canfora (2015). Se Azio fu percepita come la grande battaglia che segnò un punto di svolta nel potere di OttavianoAugusto (ad es. D.C. 51.1.1-2), la memoria di Filippi venne costantemente mantenuta viva dal regime, anche perché a Filippi, attraverso la vendetta del padre, il giovane Divifilius legittimò il proprio potere: tale continuità con Filippi si fa evidente, in particolare, nella costruzione del tempio di Marte Ultore con l'annesso Foro monumentale, che fu promesso in voto proprio a Filippi e finalmente inaugurato soltanto nel 2 a.C. (cf. Suet. Aug. 29.2 aedem Martis bello Philippensi pro ultione paterna suscepto voverat, anche $r$. gest. div. Aug. 21, p. 36 Volkm. ${ }^{3}$ in privato solo Martis Ultoris templum forumque Augustum ex manibiis feci; anche 2, p. 12 Volkm. ${ }^{3}$ ultus eorum facinus). Tutto ciò aiuta a comprendere perché, quando rievocherà la battaglia vari anni più tardi, Orazio veda Augusto stesso nel ruolo di un robusto e muscoloso eroe, unico protagonista, come una specie di dio (Marte ultore?) in terra: Epist. 2.2.47-8 arma / Caesaris Augusti non responsura lacertis (in realtà, dal punto di vista strettamente tecnicomilitare il ruolo dell'allora giovanissimo Ottaviano sul campo di Filippi fu assai modesto). 
Orazio ha voluto scrivere un carme stasiotico, sintetizzando a suo modo le allegorie nautiche del civis Alceo, per dire che era il momento, dopo tante battaglie e dissidi, di tentare un'impresa ancor più difficile, quella del 'porto della concordia', per parafrasare Quintiliano.

E dunque, che dire di Quintiliano? Che nella sostanza, possiamo ripeterlo, ha ragione: nel senso, cioè, che quella di Orazio è un'allegoria di ambito politico, che effettivamente esorta a temere i pericoli della guerra civile e a navigare verso la conciliazione. Ma, come nel caso delle Bucoliche virgiliane, il giudizio di Quintiliano va ridefinito e delimitato, perché non abbia l'effetto di paralizzare le nostre capacità interpretative, riuscendo quindi, in ultima analisi, fuorviante. $\grave{E}$ vero che Menalca, Titiro, ma a tratti anche Melibeo (e forse Coridone?), sono personaggi che alludono a fatti, sentimenti, esperienze, propri del poeta che quei personaggi concepì. Ma sarebbe assurdo stabilire una corrispondenza biunivoca tra il personaggio Menalca e l'autore Virgilio, come Quintiliano sembrerebbe voler fare nel luogo citato del libro VIII. Così, la nave di Orazio non è identificabile con la respublica, per quanto Orazio nel carme 1.14 mostri una precisa idea politica (che riguarda anche, ma indirettamente, la respublica nel suo insieme). La schematica generalizzazione navis = respublica, se poteva bastare a Quintiliano e ai suoi allievi, non può bastare agli interpreti del carme 1.14.

\section{2. 'Metafora continuata' eil testo del v. 10 (Quintiliano va qui, invece, valorizzato)}

Se, come abbiamo visto, il giudizio di Quintiliano è stato fin troppo preso sul serio per quel che riguarda l'interpretazione generale del carme, su di un punto specifico, che interessa una questione critico-testuale nel v. 10 , non se ne è tenuto abbastanza 
conto $^{19}$. Quintiliano, infatti, fa un'affermazione che avrebbe meritato di attrarre maggiore attenzione, quando dice, nello specifico, che il carme oraziano è un buon esempio del primo tipo di allegoria, in cui cioè si abbia una serie continuata di metafore (continuatis translationibus). In effetti, la navis è presentata al lettore come una 'vera nave', senza alcun esplicito riferimento a fatti extra-allegorici (cioè a quel significato 'altro' che della allegoria è la soluzione). Altrimenti, certo, gli interpreti non ne avrebbero così lungamente discusso, con divergenze e dissensi anche molto consistenti! - come abbiamo potuto vedere.

Prima di arrivare al v. 10 e alla sua questione testuale è opportuno considerare, con qualche dettaglio, la struttura del carme, proprio per comprendere come l'invenzione metaforica si articoli all'interno di esso. A ben vedere, infatti, il carme è caratterizzato da una struttura molto costruita. Lo sviluppo del tema, dunque, si divide studiatamente in tre sezioni, ciascuna con un numero decrescente di versi (il che mira probabilmente ad un effetto di progressiva condensazione e intensificazione emotiva e concettuale). Nella prima sezione, che comprende i primi dieci versi ovvero esattamente due strofe e mezzo, l'allocuzione diretta alla nave, dopo l'enfatico attacco esortativo (1-3), si dispiega nella descrizione della nave, che resta su di un piano oggettivo, puramente visuale, in corrispondenza con il verbo reggente (3 nonne vides): all'unico periodo che si estende dal v. 3 all'inizio del v. 9 seguono i quasi due versi conclusivi della sezione (9-10). Nella seconda sezione, che comprende sei versi ovvero esattamente una strofa e mezzo, viene abbandonato il modo descrittivo e la nave è presentata nella sua caratterizzazione morale tramite una vera e propria personificazione: alla nave, insomma, Orazio si rivolge come ad una persona, ribadendo

19 Riprendo qui, in una prospettiva diversa e con qualche nuova osservazione, quanto ho sostenuto in un apposito contributo critico-testuale, che, successivamente alla data del convegno, è stato nel frattempo pubblicato: Cucchiarelli (2015b). 
l'ammonizione con il conclusivo verbo cave (11-16). La terza e ultima sezione, di quattro versi ovvero una sola strofe (17-20), riprende e chiarisce il discorso morale, esprimendo l'atteggiamento del poeta nei confronti della nave e dunque specificando in senso strettamente oraziano l'allegoria. Questa tripartizione, con progressiva riduzione nel numero dei versi, va a sovrapporsi le tre parti, disuguali, sono rispettivamente di dieci, sei e quattro versi, va a sovrapporsi alla simmetrica bipartizione del carme cui già abbiamo avuto modo di fare riferimento (supra, p. 153), bipartizione dalla quale esso è suddiviso in due parti di dieci versi ciascuna (e, ciascuna, di due strofe e mezzo): la prima parte, quasi del tutto descrittiva, è assai vicina al modello alcaico (1-10); la seconda parte, morale e riflessiva, è del tutto originalmente oraziana (11-20). Sia che si guardi alla bipartizione sia che si guardi alla tripartizione, resta chiaro che tra il v. 10 e il v. 11 passa una netta incisione strutturale del carme $^{20}$. E proprio nell'ultima parola del v. 10, subito prima, dunque, di tale incisione, credo che sia da ravvisare la questione di testo che ci interessa.

Fino al v. 10 compreso, dunque, la descrizione della nave è tutta propriamente nautico-marinara. Anche gli 'dèi' non sono le divinità del mare o del cielo, che non si capisce come potrebbero essere danneggiate al pari delle vele, ma quelle immagini sacre (statuette o simulacri) che, poste in genere a poppa, proteggevano la navigazione ${ }^{21}$. Ma l'ultima parola, che chiude tutto questo drammatico movimento sintattico-descrittivo, subito a precedere la netta incisione strutturale che suddivide il carme, non ha nulla

20 Non c'è da dubitare del fatto che nel successivo v. 11 quamvis Pontica pinus eqs. inizi un nuovo periodo, secondo l'interpretazione corrente, oggi senz'altro maggioritaria tra editori e commentatori. Alcuni, invece, in passato legavano la concessiva a quel che precede.

21 Cf. Nisbet; Hubbard $(1970,185)$ ad loc., dove è osservato, appunto, che di deve riferirsi "like malus, lintea, etc., to a part of the boat"; ma già Kiessling; Heinze (1960, 73); inoltre Mayer $(2012,134)$. 
di specificamente marinaro e anzi comporta una opacizzazione dell'invenzione allegorica: a nulla servirebbe rivolgersi a quei simulacri divini ormai infranti, quando di nuovo la nave si trovasse a essere 'p r e m u t a d a $1 \mathrm{~m}$ a 1 e' (pressa ... malo). L'incolore e generico aggettivo malo, sostantivato all'ablativo singolare, rende obbligata un'interpretazione essa stessa generica di pressa che invece, di per sé, è termine del tutto proprio, e specifico, in riferimento ad un natante che si trovi ad avere cospicuamente abbassata la linea di galleggiamento (Verg., Georg. 1, 303): dopo la fiancata priva dei remi, l'albero maestro ferito, il gemito delle antenne e la carena priva dei suoi legami a rinforzo, dopo le vele e i simulacri danneggiati, la parola conclusiva del movimento stando al testo tràdito è, dunque, un generico 'male'.

Del problema dovettero in qualche misura accorgersi Nisbet, Hubbard, che, dopo aver notato la particolare semantica di pressa, osservano appunto che malo è molto meno appropriato al contesto: "In our passage the word [scil. pressa] is particularly appropriate as it is sometimes applied to the sinking of ships [...]. But malo is much less appropriate than tempestate» (1970, 185-6). Ma forse la valutazione di questo carme come un 'non-capolavoro', che ereditavano da E. Fraenkel, agevolava i due dotti e finissimi commentatori nell'ammettere in Orazio un uso linguistico meno efficace e appropriato: è vero che malo è parola generica, si potrebbe dire incolore, ma essa non può sorprendere chi veda in Orazio soprattutto una fredda e intellettualistica "determination to write allegory». Credo che qui, però, l'improprietà sia da imputare non ad Orazio ma ai manoscritti.

Credo, cioè, che l'ablativo scelto da Orazio per determinare pressa sia non malo ma salo: non un generico male opprimerebbe la nave, come potrebbe esserne oppresso un qualunque individuo (essere vivente o oggetto personificato), ma è il 'mare aperto', con il suo potente moto ondoso, ad abbassarne pericolosamente 
il galleggiamento, 'premendola giù'22. Probabile prestito dal

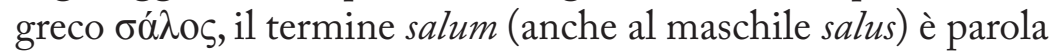
letteraria, ben presente nella lingua repubblicana, in tragedia (Enn. trag. 179 Joc.; Acc. trag. 10 R. $^{3}$ ) e nelle orazioni di Cicerone (Verr. 2.5.91; Caecin. 88) e ancora utilizzata da Livio (37.10.10; 37.13.8): con essa si indica il mare al largo, caratterizzato dalla sua forza ondosa trascinante, come ben si vede, in particolare, dal luogo di Catullo, 63.16 rapidum salum tulistis truculentaque pelagi. Il mare lontano dalla costa o quanto meno fuori dal porto (ante portum, ad esempio, nel già menzionato Liv. 37.10.10), è esattamente dove la nave si trova al momento, se il poeta deve esortarla perché nel porto, appunto, si sbrighi ad entrare (fortiter occupa portum), evitando di essere trascinata via, cioè in alto mare (in mare), dai novi fluctus. Il pericolo deve essere ribadito da un sostantivo che abbia in sé l'idea della forza trascinante del mare: e questo sostantivo, come testimonia l'esplicitazione rapidum salum di Catullo, è salo. La parola è utilizzata dallo stesso Orazio, proprio in contesto di naufragio, in un testo che con ogni verosimiglianza è anteriore al carme 1.14 (e sicuramente noto ai lettori del carme per il fatto di essere stato pubblicato in raccolta già in precedenza): non saxa nudis surdiora navitis / Neptunus alto tundit hibernus salo (Epod. 17.54-55). È ben possibile che, nel descrivere la drammatica situazione in cui si trova la nave, l'Orazio lirico si sia ricordato dell'epodo, recuperandone quella parola così significativa, con un deliberato gioco di memoria interna: in entrambi i casi salo, all'ablativo, è ultima parola del verso (e del periodo), con insistenza fonica sul suono $/ s /$ rispetto alla fine di parola precedente (voceS Salo $\approx$ bibernuS Salo). Anche Virgilio nell'Eneide, quando deve far descrivere a Ilioneo la forza travolgente del mare, che ha spinto i troiani tra secche e scogli impervi (costringendoli a riparare fortunosamente verso la riva africana) utilizza l'ablativo salo, qualificandolo con superante,

22 Mi limito qui ai dati essenziali; per una discussione filologica più dettagliata si rinvia a Cucchiarelli (2015b). 
proprio ad esprimerne la capacità soverchiante: cum subito adsurgens fluctu nimbosus Orion / in vada caeca tulit penitusque procacibus Austris / perque undas superante salo perque invia saxa I dispulit: huc pauci vestris adnavimus oris (Aen.1.535-9). Con il participio presente superante Virgilio esprime l'azione del salum sulla flotta troiana, con il participio passato pressa Orazio descrive l'effetto che la nave ne riceve.

La tradizione manoscritta, dunque, ha offuscato un tratto di lingua letteraria elevata, già repubblicana ${ }^{23}$, con cui Orazio ha voluto caratterizzare l'estremo pericolo in cui la nave versa. Una parola specifica, si potrebbe dire 'tecnica' del lessico letterario nautico, chiude con efficace vivacità la sezione, disponendosi in corrispondenza verticale con l'altrettanto specifico lintea del verso precedente ${ }^{24}$. Già nelle fasi più antiche della tradizione il relativamente difficile salo, nella sequenza pressavocessalo, dovette corrompersi con il concorso dell'aplografia in un più banale e generico malo, che sembrò scorrevole e ben comprensibile a generazioni di copisti e lettori: per chi non coglieva più in dii il riferimento ai concreti simulacri divini che corredavano la nave e quindi vedeva nel v. 10 non altro che una disperata (ma generica) invocazione a dei non più esistenti, un concetto (generico) come

${ }^{23}$ Repubblicana anche per quella sua coloritura greca che in poesia romana spesso suona 'antica' e 'poetica'. Si aggiunga che la coloritura greca è quanto mai pertinente ed evocativa, dal momento che il corrispettivo greco, da cui deriva il latino salum per prestito, figurava già nel diretto modello di Orazio, cioè Alceo (proprio, con ogni verosimiglianza, l'Alceo delle allegorie nautiche): fr. 73, $2 \mathrm{~V}$.

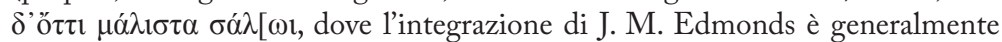
accettata da editori e studiosi; uso metaforico nel contesto della nave-città anche in Soph.Ant. 163; Oed. tyr. 24.

24 Come ha osservato Stephen Harrison nel convegno di San Paolo, la rispondenza lintea ... salo è un caso che può rientrare in uno schema oraziano piuttosto tipico (nomi specifici appartenenti alla medesima area semantica in corrispondenza verticale, collocati entrambi a fine di verso e periodo); restando al libro I dei Carmina Stephen Harrison menziona: 1.9.11-2 cupressi ... orni (nomi di alberi); 1.17.27-8 coronam ... vestem (abbigliamento); $1.21 .11-2$ pharetra ... lyra (equipaggiamento); 1.23.11-2 matrem ... viro (relazioni familiari). 
quello di 'oppresso dal male' era ben opportuno e perfettamente significativo ${ }^{25}$.

Ma qui la nave di Orazio è ancora descritta, a pieno titolo, come una vera nave, in una successione continuata di metafore, proprio come voleva Quintiliano. Una parola marinara suggella la prima sezione del carme (che lo si consideri come bipartito o come tripartito), e qui, da retore, Quintiliano ci mette sulla strada giusta: la nave di Orazio, come ogni nave, è premuta non genericamente dal male, ma dall'alto mare ${ }^{26}$.

\section{Altrenavi auguste, tra identità edissidio, sulla scia di Orazio}

Per l'interprete del carme 1,14 , che guardi ad esso come parte di una tradizione nautica (e allegorica) risalente ad Alceo e ben stabilita nella poesia ellenistica e in quella latina, è di sicura rilevanza la risposta che, sul tema della nave, danno due importanti poeti augustei, Virgilio e Ovidio. Proprio il confronto tra due forme letterarie pur così nettamente distinte, già nella percezione antica, quali la poesia lirica (Orazio) e la poesia epico-narrativa (Virgilio e Ovidio) si rivelerà utile e produttivo di significato. Ciò, almeno, è quanto ci proponiamo di mostrare in questa sezione: vorremmo, cioè, che la nostra interpretazione dell'allegoria oraziana trovasse conferma in Virgilio e Ovidio (forse, anche, ricavandone degli spunti di riflessione non inopportuni per l'opera di questi ultimi).

25 Tanto più quando si pensi che la 'pressione' del male (o del Maligno?) è concetto di forte suggestione per lettori e copisti cristiani: cfr., tra gli altri, Min. Fel. 28.4 si qui infirmior malo pressus et victus Christianum se negasset; altri esempi in Cucchiarelli (2015b, 358-9, n. 11).

26 Ancora in forma corretta, verosimilmente, il testo di Orazio era letto dall'Auctor Octaviae, che proprio dal carme 1,14 prende l'espressione salo pressa, riadattandola ad una donna, l'Agrippina ormai destinata a morte certa: ruit in pelagus rursumque salo / pressa resurgit (346-7). 
È utile, preliminarmente, ancora una breve avvertenza di metodo. Bisogna aver chiaro, cioè, che il gruppo metaforico della navigazione, nelle sue varie forme che vanno dalla comune langue alle invenzioni allegoriche dei poeti, appartiene a quel livello condiviso della cultura che attraversa tutti i mezzi espressivi, artistici e figurativi, politici e sociali. Dunque, l'interpretazione del testo poetico può soltanto giovarsi di una visione più ampia, che permetta di collocare la voce di Orazio (di Virgilio, di Ovidio...), nel contesto di pubblico che era proprio della Roma augustea ${ }^{27}$.

Dunque, anche sulla base di quanto abbiamo visto analizzando il carme 1,14 , si possono isolare due aspetti salienti della metafora-allegoria nautica, quando essa si presenti nella forma della nave personificata (naturalmente nei suoi vari gradi di personificazione): due aspetti che si riveleranno importanti anche nell'analisi di Virgilio e Ovidio.

Il primo aspetto è quello identitario, cui strettamente si collega l'autocoscienza (un aspetto, dunque, 'interno', se si vuole connaturato): la nave ricorda le proprie origini, appartiene ad un gruppo, può collaborare ad identificarlo.

Il secondo aspetto è 'esterno' rispetto alla nave, cioè il comportamento della realtà/natura, che in genere, trattandosi del mare, si esprime nei pericoli della navigazione, cui la nave (e torniamo così al primo aspetto, quello 'interno') deve rispondere con la propria capacità di sopportazione $\mathrm{o}$, se possibile, con la propria capacità previsionale.

Si capisce che i due aspetti sono di per sé legati tra loro e come tali si presentano nei testi: in un momento emotivamente

27 Sull'importanza culturale e politica di navi e navigazioni, specialmente nell'epoca di Augusto, quando alla memoria delle grandi vittorie navali repubblicane si andava sovrapponendo quella recente di Nauloco e, soprattutto, di Azio, si rinvia, in una prospettiva sostanzialmente virgiliana, a Cucchiarelli (2016). 
intenso e ricco di potenzialità poetiche (e ideologiche) come è quello della tempesta, è naturale che la nave mostri la propria identità. Nell'allegoria politica è appunto il pericolo che rivela le capacità del 'governante' e quindi la nobiltà e le qualità della nave e del suo equipaggio: d'altra parte anche per un bravo nocchiero, capace quindi di prevedere l'arrivo della tempesta, non è facile sapersi regolare di fronte ad eventi così incontrollabili, come si legge verso la conclusione di un testo politico assai importante (e ben noto a Roma almeno dai tempi di Cicerone), la settima lettera attribuita a Platone ${ }^{28}$. Nell'incontro o scontro tra le qualità dell'imbarcazione (e di chi la governa) e l'azione del mare si gioca la possibilità di successo o insuccesso, nautico e, nel caso di un testo allegorico, politico.

Nell'importante modello catulliano che già abbiamo avuto occasione di ricordare, il phaselus del carme 4 , non si può parlare di allegoria politica, naturalmente, ma i due aspetti sono ben rappresentati: è la stessa 'barchetta' a rammentare le proprie qualità marinare, in particolare l'origine dal Ponto, che le hanno permesso di superare tante difficoltà, come tanti mari e regioni (tra cui anche le isole Cicladi: 7) stanno a testimoniare, e di portare in salvo il padrone (Catullo stesso, presumibilmente: 19). È molto probabile che Orazio, guardando ad Alceo, avesse ben presente il carme catulliano: anche la sua navis proviene dai boschi del Ponto e appunto le Cicladi sono un riferimento geografico già catulliano. Ed è significativo come entrambi

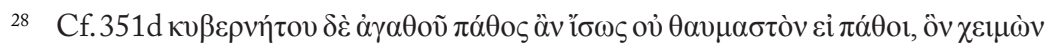

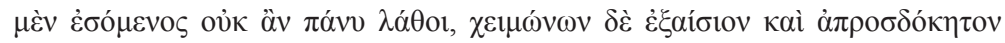

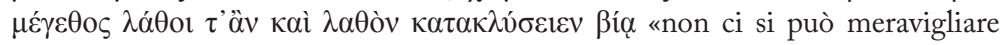
se gli succede [all'uomo buono, in particolare il buon governante, come Dione, tra i malvagi] di patire lo stesso destino del buon nocchiero, cui certo non può sfuggire l'arrivo della tempesta, ma può sfuggire invece la grandezza insolita e imprevista delle tempeste, che poi, con violenza, lo sommerge». Si ricordi già l'Alceo della nave, che osservava la necessità di saper prevedere da terra la rotta:

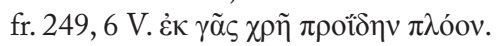


i poeti stabiliscano un vero e proprio contatto comunicativo con l'imbarcazione, che, personificata, esprime essa stessa il proprio punto di vista: in Catullo si legge ait, nel v. 2, poi nel v. 6 negat, di nuovo ait nel v. 15, dicit nel v. 16 (non sorprende che già in forma arborea il phaselus fosse piuttosto loquace: 12 loquente saepe sibilum edidit coma); in Orazio c'è un solo verbo, ma estremamente significativo, iactes (13), ad esprimere la superba 'iattanza' della nave, che appunto è così fiera di quelle nobili origini che, nella realtà, rischiano di riuscirle inutili, se non dannose.

Per il poeta lirico, attraverso la personificazione, è facile rivolgersi ad un oggetto inanimato quale un'imbarcazione è, dare ad esso la parola e quindi farsi rispondere: poeta lirico è Orazio e, da questo punto di vista, alla forma 'lirica' appartiene anche Catullo; entrambi si allineano al lirico Alceo, che aveva fatto parlare anche lui la propria nave (fr. 73, 5 V. paĩol).Il poeta epico, di per sé, ha tutto l'agio di rappresentare la nave, descriverla, raccontarne le avventure, può eventualmente rivolgersi ad essa con un'allocuzione, ma trova qualche difficoltà a farla 'parlare', dovendosi attenere nei fatti cha racconta ad un principio di realtà più stretto $\mathrm{o}$, se si preferisce, diversamente codificato. Nei poemi omerici navi e imbarcazioni si incontrano spesso, sono il vettore dei Greci e dell'avventuroso nostos di Odisseo e nel libro II dell'Iliade il catalogo ne valorizza proprio l'aspetto identitario: ad ogni flotta, ad ogni equipaggio, corrisponde un popolo. Ma in Omero non ci sono navi propriamente personificate né, tantomeno, parlanti. Fu Apollonio Rodio a trovare il modo di dare vita e parola a quella che, comunque, resta una nave molto particolare: oltre ad essere la prima nave in assoluto, Argo è diretta invenzione della dea Atena. Proprio Atena, dunque, stando alla tradizione mitografica raccolta da Apollonio, avrebbe inserito nel mezzo della chiglia di Argo una trave proveniente da una quercia di Dodona, dotata quindi di potere oracolare: per questa ragione, dunque, la stessa Argo fa sentire potentemente la 
propria voce, dando il via all'impresa, nel libro I (524-527 spec.

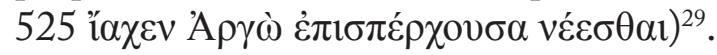

Dei limiti che il poeta epico è tenuto a rispettare, in termini di forma comunicativa e verosimiglianza, doveva ben rendersi conto Virgilio, che però riuscì anche lui a trovare un modo per dare vita e parola alle navi dell'Eneide, che sono quelle della flotta troiana. Anche Virgilio attinge alle risorse fantastiche del mito, e in particolare all'intervento divino, ma ricorre, diversamente da Apollonio, alla metamorfosi: non più una nave che ha in sé un sacro legno parlante, inserito da Atena, ma un'intera flotta che proviene da un bosco dell'Ida sacro a Cibele e che - grazie alla promessa di Giove alla stessa Cibele, infine mantenuta - si trasforma in uno stuolo di ninfe marine (9.77-122). Non la metafora, eventualmente disposta in allegoria, ma la metamorfosi è l'artifizio poetico sfruttato da Virgilio per dare parola alle navi: una forma di 'straniamento' che interviene sull'oggetto stesso e non si limita, cioè, ad agire sul livello linguistico ${ }^{30}$. Metafora e metamorfosi, dal punto di vista del poeta inventore di res e verba, condividono una analoga capacità dinamica di 'spostamento' e, quindi, di innovazione: trasformano le parole come le cose.

All'invenzione virgiliana si allineerà Ovidio, nell'ambito così appropriato di un poema interamente dedicato alle trasfor-

29 Parlerà ancora, nel quarto e ultimo libro, in una vera e propria profezia: 4.580-91. È forse significativo che le altre attestazioni di navi parlanti si abbiano, a quanto pare, nei generi comico-drammatici: nell'Argo di Eschilo (forse un dramma satiresco?), che può essere considerato un precedente diretto per Apollonio (cfr. TrGrF, III, frr. 20; 20a, pp. 135-136 Radt), e nelle Holkades di Aristofane, in cui le navi (da carico) parlavano e cantavano/danzavano perché evidentemente costituivano il coro che dà il nome alla commedia (cfr. $P C G$, III.2, frr. 415-443 K.-A.).

30 È possibile che in questa sua fantasia poetica Virgilio debba qualcosa alle invenzioni della commedia antica: si pensi al coro delle navi nelle Holkades di Aristofane, che si è appena avuto occasione di ricordare (supra, n. 29); del suo 'coro' di navi 'danzanti', Cimodocea è, evidentemente, la corifea (cfr. 224 lustrantque choreis, in corrispondenza verticale con l'apparizione del nome, alla fine del v. 225). 
mazioni, nel quale si aggiunge distintamente la funzione eziologica di spiegare il perché del mondo nelle sue varie parvenze. Vedremo come in entrambi, Ovidio e Virgilio, l'esperienza lirica della nave animata e parlante (allegorica, nel caso di Orazio), non sia passata invano ma abbia lasciato invece tracce riconoscibili. Sembra, anzi, che proprio la trovata della metamorfosi abbia permesso ai due poeti epici di recuperare quella componente di 'accortezza' (non soltanto nautica) che è o dovrebbe essere la caratteristica precipua di qualunque nave, imbarcazione o navigio, che siano o non siano allegorici.

\subsection{Lenavi di Enea in Virgilio}

Che la nave, come il carro o le armi, sia uno strumento in cui la virtù dell'eroe epico si esplica e si fa evidente, è cosa su cui non si può dubitare. Le capacità di dominio, aggressione, controllo, ma anche giustizia ed equità, che ci si può attendere da un capo, trovano dunque un loro sostituto simbolico nella nave (come in molti altri oggetti dell'apparato epico-guerriero).

Già nell'Iliade l'episodio dei giochi per Patroclo mostrava, nella dimensione agonistica ma non guerriera dell'azione ludica, come i capi dell'esercito greco sapessero misurarsi, in particolare, nella gara dei carri. Così Virgilio ha disposto nel libro $\mathrm{V}$ dell'Eneide varie gare di forza e abilità in onore di Anchise, la più cospicua delle quali è la gara delle navi. In Omero come in Virgilio i vari partecipanti danno prova nelle gare delle loro capacità (e il lettore ritrova gli eroi in azione, ognuno con le sue tipiche caratteristiche). Per quel che riguarda la gara delle navi virgiliana, non si è mancato di osservare come in essa l'abilità nel governo nautico abbia un evidente valore dimostrativo sul piano 'politico' ${ }^{31}$. Il poeta mette in condizione il lettore di riconoscere

31 La rilevanza ideologica e politica della gara delle navi è stata ben valorizzata in particolare nei lavori di Hardie (1987); Feldherr (1995); Delvigo (2001). 
nella gara delle navi valori propriamente militari e civici, che rinviano alla futura città di Roma. Per limitarci agli esempi più evidenti, già il microcatalogo delle quattro navi (5.115 quattuor ex omni delectae classe carinae) presenta navi, equipaggi e capitani come prefigurazioni, quasi si direbbe 'araldiche', di famiglie romane (in tre casi su quattro): i Memmi da Mnesteo; i Sergi da Sergesto; i Cluenzi da Cloanto (5.116-123). Altrettanto evidente il significato non esclusivamente marinaro di vicende nautiche in cui ad avere la meglio sono i capitani e gli equipaggi che riescono a restare solidali e compatti, mirando uniti al risultato: anzi, l'aver saputo coinvolgere la divinità con un'opportuna preghiera è ciò che permette a Cloanto di ottenere la vittoria (233-243). Nel caso di Sergesto, una manovra troppo audace lo porta a cozzare contro gli scogli della meta, ma egli riceve comunque un premio per aver saputo salvare la nave e i compagni (283 servatam ob navem ... sociosque reductos): una parziale analogia, dunque, con il destino del più malfamato discendente, Sergio Catilina ${ }^{32}$, ma soprattutto un contrasto, perché qui Sergesto riesce a evitare il disastro e anzi a 'salvare' i suoi.

Ma Virgilio non si limita, seguendo in sostanza l'esempio omerico, a valorizzare nei suoi capitani ed equipaggi quelle virtù (o non-virtù) che sono determinanti nel governo sia di una nave sia di un esercito o di una città. Poeta post-ellenistico, probabilmente anche stimolato dall'esperienza lirica dell'amico Orazio (l'Alceo romano), e forse in particolare proprio dal carme 1.14, Virgilio allude alla tradizione della nave-città, ma in un contesto negativo, a rimarcare le potenzialità rischiose contenute nell'immagine. L'unica nave che abbia un capitano di cui Virgilio non menzioni la discendenza romana, la Chimera di Gia, destinata a dare prova di 'malgoverno' a causa della

32 Già La Cerda notò come alla nave di Sergesto pendente dallo scoglio (5.206 inlisaque prora pependit) corrisponda la punizione di Catilina nel Tartaro, per come Virgilio la rappresenta sullo scudo di Enea in 8.668-669 et te Catilina minaci / pendentem scopulo Furiarumque ora trementem. 
lite tra lo stesso capitano e il nocchiero Menete, viene infatti paragonata proprio ad una città: ingentemque Gyas ingenti mole Chimaeram, / urbis opus, triplici pubes quam Dardana versu / impellunt, terno consurgunt ordine remi (118-120). Dimensioni enormi, grande novità tecnologica (tre ordini di remi, mentre normalmente nel mondo omerico le navi sono ad un solo ordine), notevoli potenzialità, che però risultano essere semplicemente eccessive (secondo il modello della hybris), perché disgiunte $\mathrm{da}$ un reale affiatamento nell'equipaggio e in particolare tra $\mathrm{i}$ vertici del comando, capitano e nocchiero ${ }^{33}$. Dunque, l'analogia con la città, che Virgilio evoca con la densa espressione urbis opus, resta soltanto esteriore ed è invece fallimentare sul piano della metafora politica: spinta da tanta gioventù troiana (pubes ... Dardana), la triplice Chimera non allude ad alcun futuro romano e Virgilio non nomina nessuna famiglia che si richiami al capitano $\mathrm{Gyas}^{34}$. Il poeta stimola il suo lettore a ricordarsi della metafora-allegoria che lega la nave alla città, ma soltanto perché giunga alla conclusione che qu e s t a nave epica assolutamente $\mathrm{n}$ o $\mathrm{n}$ sia paragonabile ad una città ben governata ${ }^{35}$.

La nave epica è un oggetto attraverso cui si esprime e fa evidente la capacità di comando del capo: vettore necessario al viaggio e all'azione di guerra, al tempo stesso diviene sostituto simbolico dell'autorità. Nel suo ruolo di capitano più volte viene rappresentato lo stesso Enea, con un dettaglio, quello dell'alta

33 Ad esprimere il malgoverno della città, l'analogia con una nave in cui l'equipaggio sia indisciplinato, litigioso e non rispettoso del capitano è già in Platone, rep. 6,

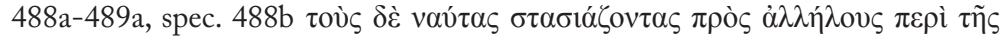

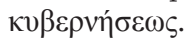

34 Sulla nave Chimera e in particolare sullespressione urbis opus rinvio a Cucchiarelli (2016, spec. 146 e n. 27).

35 Anche il timore del nocchiero Menete, che si guarda dagli "scogli nascosti» (164-165 'caeca ... / saxa timens), oltre a essere giustificato dai saxa latentia di Aen. 1.108, come osserva Delvigo (2001, 19), si spiega con l'esperienza, specificamente nautico-allegorica, della nave di Alceo, che si era trovata a

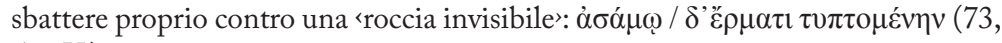
5-6 V.). 
poppa, che rappresenta con immediatezza visiva la forza della sua autorita ${ }^{36}$, e lo stesso dettaglio lo si ritrova, significativamente, per l'Augusto di Azio, nella rappresentazione celebrativa dello scudo (8.680 stans celsa in puppi). Che la poppa della nave fosse un punto focale nella percezione dell'osservatore lo conferma, a contrasto, il carme 1.14 , dove appunto proprio le puppes variopinte, adorne cioè degli emblemi più nobili e fastosi, riescono di per sé poco affidabili al marinaio timoroso: nil pictis timidus navita puppibus / fidit (14-15). Per il poeta dell'Eneide la parte posteriore della nave, dove è facile che stia l'eroe a governare la navigazione, rappresenta con la sua imponenza affidabilità e autorità; per il poeta lirico-allegorico del carme 1.14, una poppa che sia notevole soltanto per il suo aspetto, senza una reale garanzia di buon governo e forza, è null'altro che un dettaglio patetico, al più da compatire.

Particolarmente significativa in Virgilio è una scena del libro $\mathrm{X}$, in cui Enea, lungamente atteso dal figlio e dai compagni troiani, finalmente fa ritorno dall'Etruria per via di mare: giunto in contatto visivo con le sue truppe, egli appunto si mostra ritto sull'alta poppa, con lo scudo rilucente sollevato, così suscitando l'entusiasmo nell'accampamento troiano (260-2 iamque in conspectu Teucros habet et sua castra / stans celsa in puppi, clipeum cum deinde sinistra / extulit ardentem). Ma la 'poppa di Enea', secondo una formulare metonimia da cui però è appunto valorizzata proprio qu e s t a parte della nave, è apparsa al lettore già un centinaio di versi prima, quando l'eroe troiano guidava la

36 Cf. soprattutto 10.261, che citeremo presto nel testo; inoltre 4.554 Aeneas celsa in puppi iam certus eundi (Enea ha ormai deciso di staccarsi da Didone); 8.115 tum pater Aeneas puppi sic fatur ab alta (primo discorso di Enea a Evandro e agli Arcadi); anche, per Anchise, 3.527 stans celsa (v.l. prima) in puppi. Vale la pena notare che il dettaglio, del resto ricorrente nell'iconografia delle navi arcaiche, si ritrova nella nave di Enea monumentalizzata, per come essa viene descritta da Procopio di Cesarea, che afferma di averla vista con i propri occhi a Roma, nella zona dei Navalia (ma sembra assai difficile che un tale monumento esistesse già all'epoca di Virgilio): Bell. 8 (Goth. 4), 22.11; cf. Cucchiarelli (2016, 175-181). 
flotta etrusca: Aeneia puppis / prima tenet rostro Phrygios subiuncta leones, / imminet Ida super, profugis gratissima Teucris (156-158). Anche, dunque, la nave con cui Enea fa ritorno dall'Etruria è adorna di immagini estremamente significative, che rinviano al cuore dell'identità troiana (l'Ida e i leoni frigii, simbolo di Cibele, come subito vedremo), ma in questo caso l'elemento estetico, che interessa il rostro, cioè la prora dell'imbarcazione (tenet rostro), si accompagna ad una reale sovranità, di armi e carisma, ben diversamente dalle pictae puppes della nave oraziana.

Proprio l'aspetto identitario è valorizzato da Virgilio per le navi troiane e, in particolare, per la nave di Enea, a dimostrazione di come egli ne percepisse l'importanza per il tema della nave: vale la pena seguirne rapidamente lo sviluppo nei vari libri dell'Eneide. Nel libro V l'unica nave di cui non venisse prospettato l'esito romano, la Chimera, della quale anzi si enfatizzava l'origine troiana del giovane equipaggio (pubes Dardana), faceva - come abbiano visto - una riuscita modesta, mentre un soggetto troiano quanto altri mai si presentava per il vincitore, Cluenzio, cui veniva donato un ricco mantello con la raffigurazione di Ganimede sull'Ida (250-257; si noti l'enfasi su Ida data dalla ripetizione in fine dei versi 252 e 254). Già all'inizio del libro III, del resto, il poeta aveva chiarito come la flotta troiana (protagonista del poema a partire dalla tempesta con cui si apre il libro I) fosse stata costruita proprio ai piedi dell'Ida, evidentemente con il suo legname (5-6 classemque sub ipsa / Antandro et Phrygiae molimur montibus Idae), ma è soltanto con il libro IX, quando la flotta troiana è minacciata dal fuoco di Turno, che il poeta rivela i dettagli di quell'origine 'idea'. Si scopre allora che le navi provengono da un bosco dell'Ida sacro a Cibele, che appunto ha ottenuto da Giove la promessa di immortalità per le navi: la promessa si realizza a tempo debito, con la metamorfosi in ninfe marine che le sottrae alla distruzione (spec. 107-122).

Al poeta doveva talmente premere la connessione tra le navi troiane e il monte Ida, da reiterarla specificamente per la 
nave di Enea del libro X, anche a prezzo di forzare sensibilmente la coerenza del poema. La formulazione Aeneia puppis indurrebbe a credere, infatti, che questa possa essere davvero la nave che è propria di Enea (e di lui soltanto), quella cioè originale con cui è partito da Troia, e così quella raffigurazione a prora dell'Ida, 'graditissima ai Troiani esuli', che suona quanto mai appropriata per una nave 'troiana'; anche il dettaglio dei 'leoni frigii' corrisponde ad un altro elemento che, dal libro IX, il lettore ha imparato a conoscere come specifico della flotta di Enea, cioè la tutela della dea Cibele ${ }^{37}$. Ma è molto difficile che questo sia vero, che cioè l'Aeneia puppis del libro X sia l'originale nave troiana di Enea.

Partendo dalla città degli Arcadi, Enea si è recato in Etruria con un ristretto numero di accompagnatori e, soprattutto, per via di terra, con i cavalli fornitigli da Evandro: come, dunque, poteva avere con sé la sua nave, quella con cui è partito da Troia? Si aggiunga che le navi originali della flotta troiana, come si è visto, si sono trasformate in ninfe marine e a breve, anzi, si incontreranno con Enea e la sua nave, senza però che venga fatto alcun tentativo di appianare la difficoltà: la Aeneia puppis che proviene dall'Etruria si incontra con le navi troiane trasformatesi in ninfe marine, provenienti dal Lazio, e con loro si incontrano due linee narrative del testo, non perfettamente armonizzate $^{38}$. $\grave{E}$ ben possibile che Virgilio, se soltanto ne avesse

37 A breve lo stesso Enea, nel rivolgersi alla dea, ricorderà gli animali così caratteristici del suo culto e della sua iconografia: spec. 253 biiugique ad frena leones; cf. Harrison $(1991,104)$ ad 156-157.

38 In termini di «logistical problems" si esprime qui opportunamente Harrison (1991, 104); per una disamina più dettagliata, con dossografia, posso rinviare ancora a Cucchiarelli $(2016,171$, n. 73). Della questione, nella sua sostanza, si erano già resi conto i lettori antichi, come testimoniano le annotazioni di Servio (sane notatur a criticis Vergilius hoc loco, quemadmodum sic cito dixit potuisse naves Aeneae fieri: quod excusat pictura, quam solam mutatam debemus accipere) e del Servio Dan. (ergo hanc navem Aeneae ab Etruscis datam intellegamus. quidam volunt hanc navem ex his esse quibus Aeneas ad Evandrum erat evectus, et ad Etruriam terra esse portatam). 
avuto il tempo, avrebbe trovato il modo di risolvere la difficoltà, ma quel che qui conta più osservare è quanto gli premesse connotare come riconoscibilmente 'troiana' la nave con cui Enea ritorna dall'Etruria, guidando la flotta degli alleati e portando la salvezza all'accampamento. Dunque, in un momento di forte enfasi sul suo personaggio e in particolare sul suo ruolo di capo.

Questa forte istanza identitaria, che, come abbiamo visto, è tradizionale per le navi in poesia, torna immediatamente a farsi evidente nel caso delle navi troiane trasformatesi in ninfe marine. Come dice ad Enea una di esse, Cimodocea, accostandosi alla nave: nos sumus, Idaeae sacro de vertice pinus, / nunc pelagi nymphae, classis tua (230-231). Nella nuova forma, le navi della flotta troiana possono assolvere ancor meglio al loro ruolo di protezione, in questo caso non tanto contro le avversità del mare, quanto avvertendo Enea del pericolo, la durissima offensiva di Turno che ha costretto Ascanio e compagni a rinchiudersi nell'accampamento (236-240); l'esortazione ad affrettarsi è tutt'uno con una prodigiosa spinta che Cimodocea, con opportuna conoscenza tecnica (247 haud ignara modi), rivolge alla nave, qui visualizzata proprio nel suo dettaglio più 'epico' e dignitosamente rappresentativo, 1'alta poppa' (246-247 altam ...puppim $)^{39}$. In quest'ultimo gesto, con cui la ninfa favorisce la

\footnotetext{
39 La stessa trasparente etimologia del nome rinvia ad un rapporto armonico con il

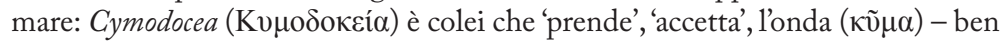
diversamente, dunque, da quel che avviene ad una nave, come quella oraziana, soggetta ai (novi) fluctus, ovvero all'amico Pompeo, trascinato in mare dall'unda della guerra civile (carm. 2.7.16). Si aggiunga che il nome è evidentemente una variazione di Cymodoce, la Nereide che figura nel corteggio marino di (propizio) accompagnamento alla flotta troiana in Aen. 5.826 (in posizione rilevata, a fine di verso e periodo); già nominata in Hom. Il. 18.39; Hes. Theog. 252-4 (dove la sua prerogativa, che condivide con Cimatoleghe, è quella di placare flutti e venti), ricomparirà in Silio Italico, che, con ogni verosimiglianza ricordandosi della Cimodocea virgiliana, la sceglierà, tra le altre Nereidi, per parlare a Proteo: 7.428-429 ad quae Cymodoce, nympharum maxima natu / Italidum: 'nosti nostros, praesage, timores eqs.' (si noti l'ormai compiuta 'italicizzazione' e il superlativo maxima, in risposta al virgiliano doctissima).
} 
navigazione di Enea (c'è dunque un contatto diretto tra la ninfa, ex nave, nata sull'Ida e la nave che porta raffigurata l'Ida), si vede come ormai la flotta troiana sia divenuta essa stessa una forza divina e benevola, tutela per i naviganti: ed Enea ben si rende conto del contesto divino, rivolgendosi, come abbiamo visto, alla divinità più appropriata, Cibele. A distanza di vari libri, con delle giunzioni non proprio perfette, si completa così il tema della 'nave dell'Ida': la costruzione all'inizio del libro III; la gara, con il primo premio contenente un soggetto ideo (Ganimede), nel libro $\mathrm{V}$; la sequenza di rischiato incendio, intervento divino, flashback esplicativo (la promessa di Giove), metamorfosi e incontro con Enea - il tutto nel segno dell'Ida e di Cibele - nei libri IX e X.

Abbiamo visto come Virgilio reinterpreti, da poeta epico, il tema della nave come depositaria di identità e al tempo stesso ausilio contro le avversità (del mare e non solo). Nel libro V, a proposito della nave Chimera, l'identità troiana (dardana) viene nominata in un contesto di imponenza (o bybris?) tecnologica, che però, in assenza di un'accorta capacità di governo, risulta fallimentare o, quanto meno, scarsamente efficace e priva di futuro romano: di conseguenza, il potenziale metaforico e allegorico insito nell'espressione urbis opus si attiva a contrasto, per significare la mancanza di una reale efficacia di governo e identitaria. Invece, nel caso delle navi idee (10.230 Idaeae ... pinus), la metamorfosi le rende eterne, divine forze benevole a sostegno di Enea in lotta per il governo dell'Italia.

Nel poema epico virgiliano, dunque, la metafora si ferma, non ha sviluppo, ma dove non arriva la metafora c'è la metamorfosi. Con un potente impulso dell'immaginazione Virgilio trova il modo di dare voce alla stessa nave, non personificandola all'interno di un gioco metaforico-allegorico, ma trasformandola in creatura parlante. Allora la nave dirà essa stessa la propria identità, che non è dal Ponto, in questo caso (come avviene in Catullo e Orazio), ma obbligatoriamente dall'Ida: su di lei, e sulle sue sorelle, adesso divenute divinità 
del mare, tutto lascia credere che potranno contare non soltanto Enea ma anche i suoi discendenti italici. Nella fase fondativa dell'Eneide nuove divinità vengono all'esistenza, una nave troiana fatta di legno sacro a Cibele diviene dea marina. Ben diversamente rispetto a quel che avviene nel dissidio civile dell'allegorica nave oraziana, che non ha più dei 'integri' su cui contare, quando, cioè, gli dei (o meglio i loro simulacri nautici) si spezzano.

Far parlare una nave 'identitaria' è stata una audacia di Virgilio, che pure non mancava almeno di una autorizzazione ellenistica, quella della prototipica nave Argo per come è rappresentata da Apollonio Rodio. Il poeta si rendeva ben conto di quale fosse la sua trovata e volle metterne a parte il lettore, richiamandone l'attenzione proprio sul tema della 'parola'. Cimodocea è presentata come 'la più dotta nell'eloquio' tra tutte le sue sorelle: quae fandi doctissima Cymodocea $(225)^{40}$ : non soltanto è una nave parlante, ma è una nave bravissima a parlare. E qui il poeta ritrova, almeno parzialmente, un altro prototipo poetico, ma romano, il Catullo del carme 4, con il suo loquace phaselus.

\subsection{Lenavi di Enea in Ovidio}

Nella sua riscrittura dell'Eneide l'Ovidio delle Metamorfosi non si lascia sfuggire l'opportunità di raccontare a modo suo la trasformazione delle navi troiane in ninfe marine, tra l'altro

\footnotetext{
40 L'atmosfera di 'sollievo' umoristico, di tutto l'episodio e in particolare del v. 225, è ben colta da Harrison (1991, 133), che cita il comm. di T. E. Page (London 1900): "fandi doctissima has been objected to as inappropriate for a sea-goddess [...], but Page's note sees the point: 'surely there is a touch of humour in the suggestion that these new-made nymphs were not yet very fluent'. Such humour matches other elements of light relief in this passage». Ma qui la notazione sulla (raffinata) capacità di espressione rimarca l'adesione ad una lunga tradizione di navi cui non mancano pensiero e parola (tradizione, in effetti, molto 'dotta': Apollonio, Catullo, Orazio; Alceo, Eschilo, Aristofane...).
} 
presentandola quasi come un ritorno ad uno stato anteriore, nel senso che sull'Ida esse avevano già avuto uno status divino (erano, insomma, già ninfe, ma arbore $\left.{ }^{41}\right)$. Particolarmente notevole, in Ovidio, il gioco immaginativo e linguistico per cui i vari dettagli delle imbarcazioni si trasformano in reali dettagli antropomorfici: colpisce, in prospettiva oraziana, la trasformazione del latus della nave (la fiancata) nel latus della fanciulla-ninfa ${ }^{42}$.

Diversamente da Virgilio, qui Ovidio non sfrutta, però, la metamorfosi per dare la possibilità alle navi di parlare: lo stile del racconto, del resto, è in Ovidio volutamente compendiario e, anzi, buona parte del divertimento per il poeta (e per il suo lettore) consiste proprio nell'assistere alla condensazione di un tema enorme quale è quello dell'Eneide. Ma anche Ovidio non trascura di valorizzare quei due aspetti che ci sono parsi fondamentali nella rappresentazione della nave in poesia (identità etnica e rapporto con i pericoli del mare): le navi ovidiane trasformate in ninfe, sebbene si trovino perfettamente a loro agio nell'elemento marino, non si scordano delle loro passate esperienze e, quindi, sono pronte ad aiutare le imbarcazioni in difficoltà, purché non trasportino gli odiati greci, responsabili del disastro troiano (met. 14.559-562 non tamen oblitae, quam multa pericula saepe / pertulerint pelago, iactatis saepe carinis / subposuere manus, nisi siqua vehebat Achivos. I cladis adhuc Phrygiae memores odere Pelasgos eqs.). Sono ninfe marine di origine troiana, ormai italiche, ma che restano ostili ai greci: soltanto, dunque, in corrispondenza della loro identità possono svolgere il loro ruolo di benevole ed esperte assistenti alla navigazione.

41 Cf. Hardie $(2015,440)$, ad met. 14.557 in montibus ortae.

42 Cf. carm. 1.14 .4 nudum remigio latus; Hardie $(2015$, 439) ad 552 latus. Va anche detto che nella tradizione della nave allegorica non mancano margini di sovrapposizione tra l'ambito politico (la nave-polis; la nave-eteria; la nave-Stato) e l'ambito erotico-femminile (la nave-donna; in special modo la nave- $\pi$ ó $\vee \eta \eta)$; se ne trova testimonianza, a quanto pare, nel commentario alcaico POxy 21, 2307 fr. 14 (306 i Voigt), sulla cui complicatissima interpretazione si rinvia a Porro $(1994,108-10)$. 


\section{Conclusione: Orazio, Quintiliano ela nave ribelle}

Passando per metafora e metamorfosi il grande tema poetico della nave dialoga tra la forma lirica e quella epica. Con la probabile mediazione di Orazio (e già di Catullo) Virgilio e Ovidio ${ }^{43}$ apprendono dalla tradizione metaforico-allegorica a leggere ed esprimere i significati ideologici della nave, che è oggetto concreto dell'esperienza e al contempo rappresentazione di valori identitari, sia individuali sia comunitari e politici.

L'allineamento di Virgilio (e, in subordine, di Ovidio) alla tradizione della nave catulliano-oraziana porta dunque con sé i due aspetti che sono tipici di quella tradizione, cioè l'identità individuale e il rapporto con gli eventi (marini e allegorici) dell'esperienza. Ovvero, se si preferisce, la personificazione della nave che figura già nel carme 1.14 di Orazio, con il suo precedente catulliano, mette in una prospettiva chiarificatrice e illumina l'invenzione epico-narrativa di Virgilio, in particolare nei suoi significati ideologici. Ma questa linea che, pur nelle sue discontinuità e difformità di forme e di contenuti, unisce Alceo, Catullo, Orazio, Virgilio e Ovidio, proprio nel suo elemento comune finisce per chiarire l'anello centrale della catena, cioè il carme 1.14. Perché nel duplice aspetto dell'identità e del rapporto con gli eventi la nave oraziana rientra sì nella lignée nautica, ma contrastivamente.

In Alceo la nave identificava il gruppo e il suo destino era tutt'uno con quello del poeta, che si sforzava, senza successo a quanto pare, di trovare una via d'uscita dalla tempesta (allegorica): al più una divergenza tra essa e il poeta potrebbe scorgersi nel fr. $73 \mathrm{~V}$., in cui la nave, provata dalle tempeste, non se la sente più di riprendere il mare, dando prova, in realtà, di avvedutezza nautica (e, c'è da credere, politica). Anche in

43 Quest'ultimo mostrerà di aver ben presente l'Alceo dei carmi nautici nella poesia dell'esilio, in particolare i Tristia; cf. Cucchiarelli (1997). 
Catullo c'è totale solidarietà tra il phaselus e il suo padrone: la barchetta, fiera della sua origine dal Ponto, racconta come sia riuscita a portarlo incolume attraverso tanti mari lontani, resistendo a venti burrascosi, per trovare quiete, infine, nella sua nuova veste di ex-voto o monumento consacrato ai Dioscuri (4, 25-26 recondita / senet quiete). Nella versione metamorfica di Virgilio le navi fanno tutt'uno con Enea e i suoi compagni e, dopo aver resistito a tempeste e vari pericoli marini e a due tentativi di incendio, finalmente, tramutate in ninfe, spiegano all'eroe attraverso la più loquace di loro come egli debba comportarsi nella guerra e ne agevolano la navigazione. Non diversamente, infine, nel poeta specialista di metamorfosi le navi troiane, memori della loro identità, restano ostili ai Greci, ma tanto più benevole, presumibilmente, verso i connazionali e i loro discendenti italici (è, in Virgilio, la mostruosa Chimera a non realizzare il suo potenziale nautico ed etnico a causa di un cattivo governo, che dunque rende soltanto dimensionale l'analogia con la città - urbis opus).

Invece, appunto, la nave di Orazio è sì evidentemente fiera della propria origine 'pontica', ma non ragiona nei termini di un'accorta saggezza nautica (almeno, in questi termini il poeta ce la presenta): ben diversamente dalla nave del fr. 73 di Alceo i pericoli del mare sembrano non scoraggiarla dal continuare $i$ suoi viaggi ed essa non ha alcuna intenzione di entrare in porto, trovando un suo quieto approdo. Non ci sta, la nave del carme 1.14, a farsi legare alla riva, per quanto malridotta sia, come appunto faceva Alceo, l'auctor stasiotico-allegorico, con la sua nave sconquassata, stando almeno alle parole di Orazio che già si è avuto occasione di citare (Carm. 1.32.7-8 iactatam religarat udo / litore navim). Né, dopo tanta devota e onorata carriera, è pronta ad acquietarsi nel porto - se non definitivamente, come aveva fatto il catulliano phaselus, almeno per un po'. La stasis qui non è una forza esterna che investa il poeta a s s i e m e alla sua nave, ma crea una frattura proprio tra il poeta e la nave: 
da strumento e principio di identità la nave si fa in Orazio protagonista del dissidio. Alceo dichiarava di non comprendere

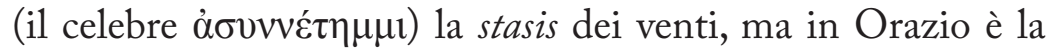
nave stessa a sottrarsi alla razionalità, ad essere incomprensibile essa stessa. La nave del carme 1.14 è tanto fiera quanto ribelle, irriducibile, impenitente. Così considerata, essa si svela come efficacissima rappresentazione di quella nobilitas romana che si ostinava a proseguire la lotta: una determinazione suicida, che però viene onorevolmente descritta dal poeta che un tempo con il destino di quella nave era stato del tutto solidale (ed è, questo, un modo abbastanza onorevole per Orazio stesso di rappresentare così il proprio distacco da quella che era stata la sua parte politica). Se proprio deve continuare a stare in mare, che almeno la navis stia attenta, dice il poeta concedendosi un ultimo moto d'affetto e preoccupazione.

È il momento di concludere, riprendendo le fila di quanto detto riguardo al carme 1.14 e alla sua interpretazione. Una prima osservazione è che, proprio nella sua incomprensibile e ribelle determinazione a proseguire la lotta con il mare, la nave di Orazio si riconferma nella sua concreta individualità. Il confronto con Catullo e, sul versante epico, con Virgilio e Ovidio aiutano a sentire l'estraneità, rispetto al testo oraziano, di una astrazione onnicomprensiva e non individuata quale è la respublica tout court: la nave poetica si collega ad un'identità precisa, a una individualità, di una persona o di un gruppo, vista in contrapposizione ad un'altra entità, individuale o collettiva. E in questo, come abbiamo visto, il carme oraziano può ritrovare la sua forza di rappresentazione poetica, che gli viene comunemente negata: nel descrivere, cioè, l'irriducibile ostinazione della nave che è quella di uno specifico e determinato equipaggio di uomini (e, certo, il poeta avrà in mente soprattutto alcuni individui a lui particolarmente vicini, come l'amico Pompeo del carme 2.7). Si comincerà ad apprezzare il carme 1.14 nel momento in cui se ne comprenderà il concreto contesto situazionale, che 
è vivo, reale, tutt'altro che astratto e intellettualisticamente costruito.

Tornando a Quintiliano, credo che una tale 'rivalutazione' del carme 1.14 di Orazio sia un caso esemplare, in cui il ripensamento di un celebre giudizio antico, canonico ormai anche per i moderni, può portare ad una più precisa esegesi e interpretazione del carme (e anche, come abbiamo visto, ad un guadagno sul piano critico-testuale). Non ci pronunciamo su questioni di estetica, che lasciamo al giudizio di ognuno. Ciascun lettore, insomma, è libero di apprezzare o non apprezzare il carme di Orazio, a seconda dei propri gusti e inclinazioni. Ma l'interprete è chiamato a chiarire il testo definendone quanto più precisamente possibile la forma comunicativa e quindi il suo significato poetico e, nel caso del carme 1.14, politico.

Per il Virgilio delle Bucoliche l'antichità ci ha trasmesso, accanto a tante elucubrazioni allegoristiche, anche il sano correttivo metodologico di Servio, che saggiamente annotava nel commento a Ecl. 1.1: hoc loco Tityri sub persona Vergilium debemus accipere; non tantum ubique, sed tantum ubi exigit ratio. Per Orazio ad aiutarci a relativizzare o se si preferisce integrare l'interpretazione di Quintiliano sono, nonostante tutte le loro ingenuità, gli antichi commentari oraziani che puntano verso i grandi 'ribelli' da cui l'azione politica del Divifilius fu contrastata. Come ogni buon maestro Quintiliano aveva in mente soprattutto il proprio programma, che nel suo caso era quello di esemplificare con chiarezza ai propri allievi il funzionamento di quel tropo che è l'allegoria. E ai nostri occhi autorevole, certo, è soprattutto Quintiliano, ben più vicino, come egli è, all'idea moderna di interprete rispetto ai modesti (o modestissimi) commentatori oraziani. Ma questi ultimi in realtà erano rimasti attaccati al testo, ben più di quel grande maestro, anche forse semplicemente perché non pretendevano di aver qualcosa d'altro da insegnare. Quintiliano è prezioso, ma va compreso, relativizzato e anche valorizzato, all'interno dei suoi propri limiti. 
Per ripetere le parole di Servio, a dirigere le nostre scelte interpretative deve essere soprattutto la ratio, che sia nutrita di conoscenza dell'autore, del suo contesto storico, dei suoi modelli letterari, che sia sgombra di preconcetti (e qui, più che i limiti di Quintiliano, tocchiamo i nostri).

\section{Bibliografia}

Anderson, W. S. 1966. Horace, Carm. 1, 14: What kind of Ship? in CPh 61: 84-98.

Canfora, L. 2015. Augusto figlio di dio. Roma-Bari: Laterza.

Clausen, W. 1994. A Commentary on Virgil, Eclogues. Oxford: Oxford University Press.

Clay,J. S. 2010. Horace and Lesbian Lyric in G. Davis (ed.), A Companion to Horace. Chicester: 128-46.

Cole, C. N. 1906. Quintilian's Quotations from the Latin Poets in $C R$ 20: 47-51.

Coleman, R. 1977. Vergil, Eclogues. Cambridge: Cambridge University Press.

Cucchiarelli, A. 1997. La nave e l'esilio (allegorie dell'ultimo Ovidio). in $M D$ 38: $215-24$.

2004. La nave e lo spettatore. Forme dell'allegoria da Alceo ad Orazio in $S I F C$ s. IV, 2: 172-88. (prima parte).

2005. La nave e lo spettatore. Forme dell'allegoria da Alceo ad Orazio in $S I F G$ s. IV, 3: 30-72 (seconda parte).

2015a. Orazio al confine del mare (tra biografia, poesia e allegoria politica) in Maia 67: 298-324.

.2015b. Il peso del male (Nota testuale a Hor. carm. 1.14.10) in RFIC 143: 354-61.

2016. Archeologia epica della nave città (in margine a Virgilio, Aen. 5,119) in Rationes Rerum, 7: 133-84.

Delvigo, M. 2001. Litus ama: linguaggio e potere nella regata virgiliana in $M D$ 47: 9-33.

Feldherr, A. 1995. Ships of State: Aeneid 5 and Augustan Circus Spectacle in ClAnt. 14: 244-65. 
Fraenkel, E. 1957. Horace, Oxford: Oxford University Press. Edizione italiana a cura di S. Lilla, con premessa di S. Mariotti: Orazio. Roma 1993. Hardie, P. 1987. Ships and ship-names in the Aeneid in Whitby, M.; Hardie, P. (eds.), Homo viator. Classical Essays for John Bramble. Bristol 1987: 163-71.

Hardie, P. 2015. Ovidio, Metamorfosi, VI (libri XIII-XV). Testo critico di R. Tarrant, traduzione di G. Chiarini. Milano: Fondazione Lorenzo Valla/Mondadori.

Harrison, S. J. 1991. Vergil, Aeneid 10. Oxford: Oxford University Press. Horsfall, N. H. 1995. Virgil: his Life and Times in N. Horsfall (ed.), $A$ Companion to the Study of Virgil, Leiden, New York, Köln: 1-24.

Kiessling, A.; Heinze, R. 1960. Q. Horatius Flaccus, Oden und Epoden. Berlin: Weidmann (10 edizione).

Knorr, O. 2006. Horace's Ship Ode (Odes 1.14) in Context: A Metaphorical Love-triangle in TAPhA 136: 149-69.

Kruschwitz, P. 2007. Fluctuat nec mergitur: Überlegungen zu Horaz' Ode 1, 14, in Hyperboreus 13: 151-73.

Lilla, S. 1993. Orazio. Con premessa di S. Mariotti. Roma.

Maltby, R. 1991. A Lexicon of Ancient Latin Etymologies. Leeds: Cairns.

Mastrocinque, A. 2016. Il muro di legno. Dall'esilio dei Focei all'Epodo XVI di Orazio in SIFC s. IV, 14: 200-9.

Mayer, R. 2012. Horace, Odes Book I. Cambridge: Cambridge University Press.

Nisbet, R. G. M.; Hubbard, M. 1970. A Commentary on Horace: Odes, Book 1, Oxford: Clarendon Press.

Odgers, M. M. 1933. Quintilian's Use of Earlier Literature in $C P h$ 28: 182-8.

Pasquali, G. 1964. Orazio lirico. Studi, a cura di A. La Penna. Firenze: Le Monnier (prima edizione: Firenze 1920).

Porro, A. 1994. Vetera Alcaica. L'esegesi di Alceo dagli Alessandrini all'età imperiale. Milano: Vita e Pensiero.

Reitzenstein, R. 1918. Die Scholien zu Horaz Od. I 14 in Nachr. Gött. Ges. Phil.-hist. Kl.: 393-6. 
Rösler, W. 1980. Dichter und Gruppe. Eine Untersuchung zu den Bedingungen und zur historischen Funktion früher griechischer Lyrik am Beispiel Alkaios. München: W. Fink.

Shackleton Bailey, D. R. 1982. Profile of Horace. London: Duckworth. Syme, R.1939. The Roman Revolution, London: Clarendon Press (edizione italiana a cura di A. Momigliano: La rivoluzione romana. Torino 1962).

Wilamowitz, U. 1913. Sappho und Simonides. Berlin: Widmann. 